\title{
Predictors and Outcomes of Cardiac Events following Thoracic Endovascular Aortic Repair in Descending Thoracic Aortic Aneurysm and Dissection
}

\author{
Derrick O. Acheampong, MD ${ }^{1} \quad$ Philip Paul, BS $^{1} \quad$ Percy Boateng, MD ${ }^{1} \quad$ I. Michael Leitman, MD ${ }^{1}$ \\ ${ }^{1}$ Department of Surgery, Icahn School of Medicine at Mount Sinai, \\ New York, New York \\ Address for correspondence I. Michael Leitman, MD, Department of \\ Surgery, Icahn School of Medicine at Mount Sinai, 1 Gustave L. Levy \\ Place, Box 1076, New York, NY 10029 \\ AORTA 2020;8:6-13. \\ (e-mail: Michael.leitman@mssm.edu).
}

\section{Abstract \\ Keywords \\ - cardiac events \\ - TEVAR \\ - aortic aneurysm \\ - risk factors \\ - endovascular \\ - ACS-NSQIP}

Background Cardiac events following thoracic endovascular aortic repair (TEVAR) have been associated with significant morbidity and mortality. However, predictors of post-TEVAR cardiac events in descending thoracic aortic aneurysm or dissection are poorly understood.

Methods A retrospective analysis of completed TEVAR procedures performed from 2010 to 2016 was conducted using the ACS-NSQIP (American College of Surgeons National Surgical Quality Improvement Program) participant user file database. Adult patients ( $\geq 18$ years) who underwent TEVAR for descending thoracic aortic aneurysm or dissection were identified and 30-day outcomes were examined. An initial univariate analysis was performed to determine associations between all patient variables and cardiac events, defined as myocardial infarction or cardiac arrest that occurred $\leq 30$ days of surgery. Multivariate logistic regression was subsequently performed to identify independent risk factors for cardiac events following TEVAR.

Results The study identified 150 out of 2,905 (5.2\%) patients who underwent TEVAR for descending thoracic aortic aneurysm or dissection who developed cardiac events. No significant difference in incidence of cardiac events was noted among patients presenting with aortic aneurysm or dissection $(p=0.339)$. The overall 30-day mortality rate for all patients was $9.1 \%$. Independent preoperative predictors of post-TEVAR cardiac events included emergency procedure (odds ratio [OR] 2.80, 95\% confidence interval [CI] 1.9-4.1, $p<0.01$ ); American Society of Anesthesiologists score $>3$ (OR 1.71, 95\% Cl 1.1-2.6, $p=0.01$ ), ventilator dependence (OR 2.33, 95\% Cl 1.3-4.2, $p<0.01)$, renal failure (OR 2.53, 95\% Cl 1.50-4.3, $p<0.01$ ), blood transfusion (OR 1.84, 95\% Cl 1.1-3.2, $p=0.03$ ), and preoperative leukocytosis (OR 2.45, 1.6-3.8, $p<0.01$ ). After TEVAR, unplanned reintubation (OR 5.52, 95\% Cl 3.5-8.8, $p<0.01$ ), prolonged mechanical ventilation (OR 1.94, $95 \% \mathrm{Cl} 1.2-3.2, p=0.011$ ), and postoperative blood transfusion (OR 4.02, $95 \% \mathrm{Cl}$ $2.70-6.0, p<0.01)$ were independent predictors of cardiac events. Cardiac events greatly increased mortality (60.7 vs. $5.5 \%$ ), total length of hospital stay $(13.2 \pm 14.7$ days vs. $8.3 \pm 9.3$ days), and readmission rates (19.3 vs. $8.2 \%, p<0.01$ ).

Conclusions Cardiac events following TEVAR are associated with significant mortality. Patients with these risk factors should be appropriately monitored to improve outcomes. received

May 1, 2018

accepted after revision

November 18, 2019
DOI https://doi.org/

$10.1055 / \mathrm{s}-0040-1701606$ ISSN 2325-4637.
Copyright $\odot 2020$ by Thieme Medical Publishers, Inc., 333 Seventh Avenue, New York, NY 10001, USA Tel: +1(212) 760-0888
License terms

(c) $($ i) 


\section{Introduction}

Thoracic endovascular aortic repair (TEVAR) is becoming a preferable approach for treating thoracic aortic pathologies due to its reduced perioperative morbidity and mortality. Nonetheless, major postoperative complications have been reported. This study identified risk factors for cardiac events (CEs), defined as either myocardial infarction (MI) or cardiac arrest $\leq 30$ days following TEVAR in descending thoracic aortic aneurysm or dissection. Understanding these risk factors should allow for appropriate resources to be given to at-risk patients to improve morbidity and mortality following TEVAR.

Cardiac events continue to remain a significant cause of morbidity and mortality following TEVAR. ${ }^{1,2}$ This makes it necessary to determine risk prediction models that would allow surgeons to identify at-risk patients to provide appropriate perioperative management that would minimize morbidity and improve post-TEVAR outcomes. The present study aims to identify risk factors for post-TEVAR CEs in descending thoracic aortic aneurysm or dissection to allow for optimal management of at-risk patients. It is our hope that by providing a practical risk assessment model, surgeons will have a better insight and understanding of at-risk patients to allow for more targeted perioperative management that might lead to improvement of outcomes.

\section{Materials and Methods}

\section{Data Source and Acquisition}

Data were retrospectively extracted from the ACS-NSQIP (American College of Surgeons National Surgical Quality Improvement Program) database from 2010 to 2016. The ACS-NSQIP is a large risk-adjusted database that was originally created in 1994 to provide 30-day surgical outcomes from the Veteran Affairs Healthcare Systems. ${ }^{3}$ Currently, the database comprises over 670 hospitals around the world, with patient data collected by trained nurse reviewers. Overall, the database includes over 150 preoperative and intraoperative variables and 30-day postoperative outcomes.

\section{Disclaimer}

The American College of Surgeons National Surgical Quality Improvement Program and the hospitals participating in the ACS-NSQIP are the source of the data used herein; they have not verified and are not responsible for the statistical validity of the data analysis or the conclusions derived by the authors.

\section{Inclusion Criteria}

Adult patients, 18 years and older, who underwent TEVAR were identified using current procedural terminology (CPT) codes 33880, 33881, 33883, 33886, 33889, 33891. Patients included for analyses met the following criteria: (1) indication for TEVAR was for aortic pathologies of descending thoracic aortic aneurysm or dissection, and (2) postoperative outcome variable for MI and cardiac arrest was documented and not missing. Aortic dissections could not be subgrouped into Stanford Type A or Type B since this information is not provided in the ACS-NSQIP database.
Cardiac arrest was defined as the absence of cardiac rhythm or the presence of chaotic cardiac rhythm that results in loss of consciousness requiring the initiation of any component of basic and/or advanced cardiac life support. Myocardial infarction was defined as a new transmural acute MI occurring during surgery or within 30 days following surgery as manifested by new Q-waves on ECG (electrocardiogram). ${ }^{4}$

\section{Variable Definition}

Patient demographics included age, gender, and race (white, black, Asian, other); other race was made up of American Indians, Alaska Native, Native Hawaiian, Pacific Islander, and unknown or not reported. Other preoperative variables analyzed included body mass index, American Society of Anesthesiologists (ASA) score classification, emergency surgery, smoking (within 1 year of surgery), alcohol abuse (at least 2 drinks per day), dyspnea (at rest or with moderate exertion) $\leq 30$ days prior to surgery, wound infection, diabetes (noninsulin dependent or insulin dependent), dependent functional health status (partially or totally dependent) $\leq 30$ days prior to surgery, ventilator dependence $\leq \mathbf{4 8}$ hours prior to surgery, history of chronic obstructive pulmonary disease $\leq 30$ days prior to surgery, pneumonia, congestive heart failure, MI, angina, previous cardiac surgery or percutaneous coronary intervention, hypertension requiring medications, renal failure (acute renal failure and dialysis), transient ischemic attack, cerebral vascular accident (with or without neurological deficits), hemiplegia or paraplegia, tumor or brain occupying lesions in the central nervous system, disseminated cancer, wound infection, weight loss ( $\geq 10 \%$ of body weight in last six months), steroid use, bleeding disorder (which includes hypothrombotic conditions such as Vitamin K deficiencies, hemophilia, thrombocytopenia, and long-term anticoagulant use, though aspirin is not included within this categorization), blood transfusion of whole/packed red blood cells (PRBCs) in 72 hours prior to surgery, prior operation within 30 days, and systemic sepsis (systemic inflammatory response syndrome [SIRS], sepsis, and septic shock).

Available preoperative laboratory variables analyzed included serum sodium, blood urea nitrogen (BUN), creatinine, albumin, total bilirubin, aspartate aminotransferase (serum glutamic-oxaloacetic transaminase [SGOT]), alkaline phosphatase, white blood count (WBC), hematocrit, platelet count, prothrombin time, partial thromboplastin time, and international normalized ratio (INR). Intraoperative variables included operative and anesthesia times.

Thirty-day postoperative adverse outcomes as predictors of CEs following TEVAR were also analyzed. These included pneumonia, unplanned reintubation, pulmonary embolism, prolonged mechanical ventilation ( $>48$ hours following surgery), renal complications (progressive renal insufficiency, acute renal failure, urinary tract infection), neurological complications (stroke or cerebral vascular accidents, coma $>24$ hours, peripheral nerve injury), packed RBC transfusions, deep venous thrombosis (DVT), surgical site infection, wound disruption, systemic sepsis, and unplanned return to the operating room.

The associations between CEs and mortality, readmission, and total length of hospital stay were also analyzed. 


\section{Statistical Analysis}

Statistical analyses were performed using SPSS software (Version 22, IBM Corporation, Chicago, IL). Descriptive analysis was reported as mean \pm standard deviation for continuous data, and percentages for categorical data. Patients were grouped into those who experienced CEs and those who did not, and the risk factors were analyzed. Univariate analysis was performed using chi-square tests to correlate baseline preoperative, intraoperative, and postoperative risk factors with post-TEVAR CEs. All variables that had a $p$-value of less than 0.05 in the univariate analysis were subsequently included in the final multivariate logistic regression with CEs as the dependent variable.

This study was reviewed and approved by the Icahn School of Medicine at Mount Sinai Institutional Review Board.

\section{Results}

The present study identified a total of 2,905 patients who underwent TEVAR from 2010 to 2016, 150 (5.2\%) of whom experienced CEs. The overall 30-day mortality rate for all patients was $9.1 \%$. Patients who experienced post-TEVAR CEs had a significantly high incidence of mortality (60.7 vs. $5.5 \%$, $p<0.01)$ than those who did not. TEVAR was performed more frequently in descending thoracic aortic aneurysm than dissection (64.3 vs. $35.7 \%$ ). There was no significant difference in the incidence of TEVAR among aortic aneurysm or dissection patients $(p=0.339)$. Of the patients who experienced CEs post-TEVAR, 67.3\% presented with aortic aneurysm compared with $32.7 \%$ with dissection.

-Table 1 summarizes univariate analysis for patient demographics, comorbidities, and perioperative factors on risks of CEs. ASA $>3$, emergency surgery, dependent functional status, preoperative ventilator dependence, renal failure, bleeding disorder, blood transfusion, systemic sepsis, BUN $\geq 23 \mathrm{mg} / \mathrm{dL}$, creatinine $\geq 1.2$, albumin $<3.5 \mathrm{mg} / \mathrm{dL}$, total bilirubin $>1.2 \mathrm{mg} / \mathrm{dL}$, SGOT $>35 \mathrm{U} / \mathrm{L}$, alkaline phosphate $>126 \mathrm{U} / \mathrm{L}, \mathrm{WBC}>11 \mathrm{~K} / \mathrm{uL}$, hematocrit $<34 \%$, INR $>1.5$, and operative time $>180$ minutes were significantly associated with CEs following TEVAR.

Following TEVAR, postoperative pneumonia, unplanned reintubation, prolonged mechanical ventilation, renal complications, neurological complications, blood transfusions, DVT, systemic sepsis, and unplanned return to odds ratio (OR) were found to be significantly associated with CEs after univariate analysis

Multivariate logistic regression analysis confirmed that ASA $>3$ (OR 1.71, 95\% confidence interval [CI] 1.11-2.63, $p=0.01$ ), emergency surgery (OR 2.80, 95\% CI 1.91-4.12, $p<0.01$ ), ventilator dependence (OR 2.33, 95\% CI 1.30-4.17, $p<0.01$ ), preoperative renal failure (OR $2.53,95 \%$ CI 1.49-4.31, $p<0.01$ ), preoperative (OR 1.84, 95\% CI 1.07-3.16, $p=0.03$ ), and postoperative (OR 4.02, 95\% CI 2.69-6.01, $p<0.01$ ) blood transfusion, WBC $>11 \mathrm{~K} / \mathrm{uL}$ (OR 2.45, 1.57-3.84, $p<0.01$ ), postoperative prolonged mechanical ventilation (OR 1.94, 95\% CI 1.17-3.24, $p=0.011$ ), and unplanned reintubation (OR 5.52, 95\% Cl 3.46$8.82, p<0.01$ ) were significantly and independently associated with post-TEVAR CEs ( - Table 2 ).
Overall, patients who experienced CEs following TEVAR were more likely to have prolonged hospitalization $(13.16 \pm 14.72$ vs. $8.3 \pm 9.32$ days, $p<0.01)$ and higher readmission rates (19.3 vs. $8.2 \%, p<0.01 ;-$ Table 3 ).

\section{Discussion}

Prior studies have evaluated risk factors for CEs in patients undergoing major vascular surgery ${ }^{5,6}$; however, there are scarce data that provide information on perioperative risk factors for CEs in TEVAR patients. In fact, the only available study that provides cardiac risk stratification in TEVAR patients was one by Ganapathi et al ${ }^{1}$ which reported on only preoperative risk factors for CEs following TEVAR at a single institution. To the best of our knowledge, our study thus becomes the first multicenter study to present a perioperative risk prediction model that allows for identifying patients at highest risk of CEs following TEVAR. In evaluating risk factors for post-TEVAR CEs, it is essential to appreciate the burden that CEs place on surgeons, patients, and the health care system. In our present study, CEs were associated with high rates of mortality, readmission, and prolonged hospitalization.

Overall, this was a large ACS-NSQIP study of 2,905 patients who underwent TEVAR for descending thoracic aortic aneurysm or dissection, of whom 150 (5.2\%) experienced CEs. This $5.2 \%$ incidence of CEs is comparable with published results. ${ }^{1,2}$ Of the 150 patients who experienced CEs, $67.3 \%$ presented with descending thoracic aortic aneurysm compared with $32.7 \%$ with dissection. However, there were no significant differences for incidence of post-TEVAR CEs between aneurysm and dissection patients. This study's overall 30-day mortality rate of $9.1 \%$, which is similar to other reported studies, ${ }^{7-9}$ suggests decreased mortality compared with open surgery. In-hospital mortality for open repair of descending thoracic aortic aneurysms and/or dissections has been reported as high as $32.1 \%{ }^{7,10}$

Our analysis suggests that ASA $>3$, emergency surgery, dependent functional status, preoperative renal failure, leukocytosis, unplanned reintubation, and perioperative blood transfusion were independently associated with post-TEVAR CEs. After TEVAR, unplanned reintubation was an associated significant and independent risk factor.

These findings are perhaps not surprising. Generally, patients who undergo emergency major vascular surgery usually present with life-threatening conditions ${ }^{11}$ that make them more susceptible to develop postoperative CEs. There is also evidence that patients who are functionally dependent have a greater medical burden, both acutely and chronically. ${ }^{12}$ Scarborough et $\mathrm{al}^{12}$ observed that among patients who underwent complex general or vascular surgery, functional dependency was independently associated with postoperative morbidity and mortality. Crawford et $\mathrm{al}^{13}$ also observed that preoperative functional dependence is associated with all adverse postoperative complications in patients who underwent infrainguinal surgical bypass.

Similarly, preoperative renal failure has been associated with poor outcomes following major vascular and endovascular surgery. ${ }^{14}$ Patients in renal failure often have significant 
Table 1 Univariate analysis of patient demographics, comorbidities, and perioperative factors on the risk of cardiac events following thoracic endovascular aortic repair

\begin{tabular}{|c|c|c|c|c|c|}
\hline Variables & $\begin{array}{l}\text { Cardiac } \\
\text { events } \\
N=150\end{array}$ & $\begin{array}{l}\text { \% Cardiac } \\
\text { events }\end{array}$ & $\begin{array}{l}\text { No Cardiac } \\
\text { events } \\
N=2,755\end{array}$ & $\begin{array}{l}\text { \% No Cardiac } \\
\text { events }\end{array}$ & $p$-Value \\
\hline \multicolumn{6}{|l|}{ Demographics: } \\
\hline Age in years (mean $\pm S D)$ & \multicolumn{2}{|c|}{$68.9 \pm 12.93$} & \multicolumn{2}{|l|}{$68.1 \pm 13.02$} & 0.498 \\
\hline BMI in $\mathrm{kg} / \mathrm{m}^{2}($ mean \pm SD $)$ & \multicolumn{2}{|c|}{$28.2 \pm 6.83$} & \multicolumn{2}{|l|}{$28.7 \pm 6.60$} & 0.38 \\
\hline \multicolumn{6}{|l|}{ Gender: } \\
\hline Male & 79 & $52.7 \%$ & 1,584 & $57.5 \%$ & \multirow[t]{2}{*}{0.248} \\
\hline Female & 71 & $47.3 \%$ & 1,171 & $42.5 \%$ & \\
\hline \multicolumn{6}{|l|}{ Race: } \\
\hline Black & 36 & $24.0 \%$ & 499 & $18.1 \%$ & \multirow[t]{4}{*}{0.290} \\
\hline White & 96 & $64.0 \%$ & 1,898 & $68.9 \%$ & \\
\hline Asian & 4 & $2.7 \%$ & 102 & $3.7 \%$ & \\
\hline Other/Unknown & 14 & $9.3 \%$ & 256 & $9.3 \%$ & \\
\hline $\mathrm{BMI}>30 \mathrm{~kg} / \mathrm{m}^{2}$ & 45 & $30.0 \%$ & 951 & $34.5 \%$ & 0.260 \\
\hline Age $>65 y$ & 99 & $66.0 \%$ & 1,810 & $65.7 \%$ & 0.932 \\
\hline \multicolumn{6}{|l|}{ Comorbidities: } \\
\hline ASA score $>3$ & 117 & $78.0 \%$ & 1,438 & $52.2 \%$ & $<0.01^{\mathrm{a}}$ \\
\hline Emergency procedure & 80 & $53.3 \%$ & 543 & $19.7 \%$ & $<0.01^{a}$ \\
\hline Tobacco smoking & 48 & $32.0 \%$ & 876 & $31.8 \%$ & 0.966 \\
\hline $\mathrm{ETOH}$ & 105 & $0.7 \%$ & 19 & $0.7 \%$ & 0.948 \\
\hline Dyspnea & 27 & $18.0 \%$ & 504 & $18.3 \%$ & 0.919 \\
\hline Dependent functional status & 19 & $12.7 \%$ & 149 & $5.4 \%$ & $<0.01^{a}$ \\
\hline Diabetes & 19 & $12.7 \%$ & 347 & $12.6 \%$ & 0.980 \\
\hline Ventilator dependence & 24 & $16.0 \%$ & 69 & $2.5 \%$ & $<0.01^{a}$ \\
\hline COPD & 29 & 19.3 & 460 & 16.7 & 0.394 \\
\hline Pneumonia & 0 & $0 \%$ & 11 & $0.4 \%$ & 0.738 \\
\hline $\mathrm{CHF}$ & 4 & $2.7 \%$ & 77 & $2.8 \%$ & 0.906 \\
\hline Myocardial infarction & 2 & $1.3 \%$ & 13 & $0.5 \%$ & 0.362 \\
\hline Previous cardiac surgery or $\mathrm{PCl}$ & 13 & $8.7 \%$ & 212 & $7.7 \%$ & 0.752 \\
\hline Angina & 3 & $2.0 \%$ & 35 & $1.3 \%$ & 0.659 \\
\hline Hypertension & 126 & $84.0 \%$ & 2325 & $84.4 \%$ & 0.898 \\
\hline Peripheral vascular disease & 5 & $3.3 \%$ & 52 & $1.9 \%$ & 0.411 \\
\hline Renal failure & 22 & $14.7 \%$ & 132 & $4.8 \%$ & $<0.01^{a}$ \\
\hline TIA & 2 & $1.3 \%$ & 41 & $1.5 \%$ & 0.941 \\
\hline CVA with or without neurological deficits & 2 & $1.3 \%$ & 77 & $2.8 \%$ & 0.551 \\
\hline Hemiplegia/Paraplegia & 3 & $2.0 \%$ & 16 & $0.6 \%$ & 0.109 \\
\hline Tumor or brain occupying lesion in CNS & 0 & $0 \%$ & 3 & $0.1 \%$ & 0.902 \\
\hline Disseminated cancer & 1 & $0.7 \%$ & 22 & $0.8 \%$ & 0.825 \\
\hline Wound infection & 6 & $4.0 \%$ & 50 & $1.8 \%$ & 0.052 \\
\hline Steroid use & 11 & $7.3 \%$ & 143 & $5.2 \%$ & 0.245 \\
\hline Weight loss & 4 & $2.7 \%$ & 55 & $2.0 \%$ & 0.595 \\
\hline Bleeding disorder & 31 & $20.7 \%$ & 314 & $11.4 \%$ & $<0.01^{\mathrm{a}}$ \\
\hline Blood transfusion & 28 & $18.7 \%$ & 110 & $4.0 \%$ & $<0.01^{a}$ \\
\hline
\end{tabular}


10 Cardiac Events after Thoracic Endovascular Aortic Repair Acheampong et al.

Table 1 (Continued)

\begin{tabular}{|c|c|c|c|c|c|}
\hline Variables & $\begin{array}{l}\text { Cardiac } \\
\text { events } \\
N=150\end{array}$ & $\begin{array}{l}\text { \% Cardiac } \\
\text { events }\end{array}$ & $\begin{array}{l}\text { No Cardiac } \\
\text { events } \\
N=2,755\end{array}$ & $\begin{array}{l}\text { \% No Cardiac } \\
\text { events }\end{array}$ & $p$-Value \\
\hline Systemic sepsis & 38 & $25.3 \%$ & 231 & $8.4 \%$ & $<0.01^{\mathrm{a}}$ \\
\hline Surgery within $30 \mathrm{~d}$ of TEVAR & 3 & $2.0 \%$ & 61 & $2.2 \%$ & 0.879 \\
\hline \multicolumn{6}{|l|}{ Preoperative laboratory: } \\
\hline Sodium $<135 \mathrm{mEg} / \mathrm{L}$ & 24 & $16.0 \%$ & 328 & $11.9 \%$ & 0.326 \\
\hline Blood urea nitrogen $\geq 23 \mathrm{mg} / \mathrm{dL}$ & 57 & $38.0 \%$ & 769 & $27.9 \%$ & $0.012^{\mathrm{a}}$ \\
\hline Creatinine $\geq 1.2 \mathrm{mg} / \mathrm{dL}$ & 68 & $45.3 \%$ & 900 & $32.7 \%$ & $<0.01^{\mathrm{a}}$ \\
\hline Albumin $<3.5 \mathrm{mg} / \mathrm{dL}$ & 74 & $49.3 \%$ & 858 & $31.1 \%$ & $<0.01^{\mathrm{a}}$ \\
\hline Total bilirubin $>1.2 \mathrm{mg} / \mathrm{dL}$ & 14 & $9.3 \%$ & 151 & $5.5 \%$ & $<0.01^{\mathrm{a}}$ \\
\hline SGOT $>35 \mathrm{U} / \mathrm{L}$ & 22 & $14.7 \%$ & 224 & $8.1 \%$ & $<0.01^{\mathrm{a}}$ \\
\hline Alkaline phosphate $>126 \mathrm{U} / \mathrm{L}$ & 15 & $10.0 \%$ & 184 & $6.7 \%$ & $0.015^{\mathrm{a}}$ \\
\hline $\mathrm{WBC}>11 \mathrm{~K} / \mathrm{uL}$ & 60 & $40.0 \%$ & 471 & $17.1 \%$ & $<0.01^{\mathrm{a}}$ \\
\hline WBC $<4.5 \mathrm{~K} / \mathrm{uL}$ & 6 & $4.0 \%$ & 116 & $4.2 \%$ & 0.992 \\
\hline Hematocrit $<34 \%$ & 72 & $48 \%$ & 857 & $31.11 \%$ & $<0.01^{\mathrm{a}}$ \\
\hline Platelet $<150 \mathrm{~K} / \mathrm{uL}$ & 28 & $18.7 \%$ & 445 & $16.2 \%$ & 0.640 \\
\hline PTT $>35 \mathrm{~s}$ & 22 & $14.7 \%$ & 426 & $15.5 \%$ & 0.170 \\
\hline PT $>15 s$ & 11 & $7.3 \%$ & 118 & $4.3 \%$ & 0.189 \\
\hline INR $>1.5$ & 14 & $9.3 \%$ & 139 & $5.1 \%$ & $0.027^{a}$ \\
\hline \multicolumn{6}{|l|}{ Perioperative variables: } \\
\hline \multicolumn{6}{|l|}{ Pathology type } \\
\hline Aneurysm & 101 & $67.3 \%$ & 1763 & $64.0 \%$ & \multirow[t]{2}{*}{0.339} \\
\hline Dissection & 49 & $32.7 \%$ & 992 & $36.0 \%$ & \\
\hline \multicolumn{6}{|l|}{ Intraoperative variables: } \\
\hline Anesthesia time $>300 \mathrm{~min}$ & 15 & $10.0 \%$ & 218 & $7.9 \%$ & 0.220 \\
\hline Operation time $>180 \mathrm{~min}$ & 79 & $52.7 \%$ & 827 & $30.0 \%$ & $<0.01^{\mathrm{a}}$ \\
\hline \multicolumn{6}{|l|}{ Postoperative variables: } \\
\hline Pneumonia & 29 & $19.3 \%$ & 129 & $4.7 \%$ & $<0.01^{\mathrm{a}}$ \\
\hline Unplanned reintubation & 58 & $38.7 \%$ & 140 & $5.1 \%$ & $<0.01^{\mathrm{a}}$ \\
\hline Pulmonary embolism & 1 & $0.7 \%$ & 11 & $0.4 \%$ & 0.619 \\
\hline Prolonged mechanical ventilation & 56 & $37.3 \%$ & 198 & $7.2 \%$ & $<0.01^{\mathrm{a}}$ \\
\hline Renal complications & 28 & $18.7 \%$ & 146 & $5.3 \%$ & $<0.01^{\mathrm{a}}$ \\
\hline Neurological complications & 18 & $12.0 \%$ & 107 & $3.9 \%$ & $<0.01^{\mathrm{a}}$ \\
\hline Occurrences of bleeding transfusions & 100 & $66.7 \%$ & 678 & $24.6 \%$ & $<0.01^{\mathrm{a}}$ \\
\hline DVT & 8 & $5.3 \%$ & 41 & $1.5 \%$ & $<0.01^{\mathrm{a}}$ \\
\hline Surgical site infection & 2 & $1.3 \%$ & 47 & $1.7 \%$ & 0.708 \\
\hline Wound disruption & 0 & $0 \%$ & 5 & $0.2 \%$ & 0.567 \\
\hline Systemic sepsis & 19 & $12.7 \%$ & 107 & $3.9 \%$ & $<0.01^{\mathrm{a}}$ \\
\hline URTOR & 34 & $22.7 \%$ & 215 & $7.8 \%$ & $<0.01^{\mathrm{a}}$ \\
\hline
\end{tabular}

Abbreviations: ASA, American Society of Anesthesiologists; BMI, body mass index; CHF, congestive heart failure; CNS, central nervous system; COPD, chronic obstructive pulmonary disease; CVA, cerebral vascular accidents; DVT, deep venous thrombosis; INR, international normalized ratio; PCI, percutaneous coronary intervention; PT, prothrombin time; PTT, partial thromboplastin time; SD, standard deviation; SGOT, serum glutamicoxaloacetic transaminase; TEVAR, thoracic endovascular aortic repair; TIA, transient ischemic attack; URTOR, unplanned return to operating room; WBC, white blood count.

${ }^{a}$ Significant $p$-values that were included in multivariate logistic regression analysis. 
Table 2 Multivariable logistic regression identifying predictors of cardiac events following thoracic endovascular aortic repair

\begin{tabular}{|c|c|c|c|c|}
\hline \multirow[t]{2}{*}{ Outcome } & \multirow[t]{2}{*}{ Odds ratio } & \multicolumn{2}{|c|}{$\mathrm{Cl}$ interval (95\%) } & \multirow[t]{2}{*}{$p$-Value } \\
\hline & & Lower bound & Upper bound & \\
\hline ASA $>3$ & 1.710 & 1.113 & 2.627 & 0.014 \\
\hline Emergency case & 2.804 & 1.909 & 4.118 & $<0.01$ \\
\hline Dependent functional status & 1.375 & 0.786 & 2.405 & 0.265 \\
\hline Ventilator dependence & 2.326 & 1.296 & 4.174 & $<0.01$ \\
\hline Renal failure & 2.534 & 1.491 & 4.308 & $<0.01$ \\
\hline Bleeding disorder & 1.514 & 0.973 & 2.356 & 0.066 \\
\hline Blood transfusion & 1.840 & 1.073 & 3.155 & 0.027 \\
\hline Systemic sepsis & 1.405 & 0.891 & 2.216 & 0.143 \\
\hline Blood urea nitrogen $\geq 23 \mathrm{mg} / \mathrm{dL}$ & 1.006 & 0.594 & 1.705 & 0.981 \\
\hline Creatinine $\geq 1.2 \mathrm{mg} / \mathrm{dL}$ & 1.489 & 0.898 & 2.469 & 0.122 \\
\hline Albumin $<3.5 \mathrm{mg} / \mathrm{dL}$ & 1.538 & 0.953 & 2.482 & 0.078 \\
\hline Total bilirubin $>1.2 \mathrm{mg} / \mathrm{dL}$ & 1.178 & 0.605 & 2.295 & 0.630 \\
\hline SGOT $>35 \mathrm{U} / \mathrm{L}$ & 1.246 & 0.720 & 2.155 & 0.432 \\
\hline Alkaline phosphate $>126 \mathrm{U} / \mathrm{L}$ & 1.079 & 0.575 & 2.025 & 0.813 \\
\hline WBC $>11 \mathrm{~K} / \mathrm{uL}$ & 2.452 & 1.567 & 3.835 & $<0.01$ \\
\hline Hematocrit $<34 \%$ & 1.025 & 0.650 & 1.616 & 0.915 \\
\hline INR $>1.5$ & 1.737 & 0.878 & 3.438 & 0.113 \\
\hline Operative time $>180 \mathrm{~min}$ & 0.958 & 0.401 & 2.288 & 0.922 \\
\hline Pneumonia & 1.275 & 0.718 & 2.265 & 0.407 \\
\hline Unplanned reintubation & 5.520 & 3.455 & 8.820 & $<0.01$ \\
\hline Prolonged mechanical ventilation & 1.943 & 1.166 & 3.240 & 0.011 \\
\hline Renal complications & 1.564 & 0.875 & 2.796 & 0.131 \\
\hline Neurological complications & 1.209 & 0.634 & 2.306 & 0.565 \\
\hline Bleeding transfusions & 4.018 & 2.685 & 6.013 & $<0.01$ \\
\hline DVT & 1.733 & 0.693 & 4.334 & 0.240 \\
\hline Sepsis & 0.746 & 0.376 & 1.477 & 0.400 \\
\hline URTOR & 1.094 & 0.518 & 2.311 & 0.815 \\
\hline
\end{tabular}

Abbreviations: ASA, American Society of Anesthesiologists; Cl, confidence interval; DVT, deep venous thrombosis; INR, international normalized ratio; OR, odds ratio; SGOT, aspartate aminotransferase; TEVAR, thoracic endovascular aortic repair; URTOR, unplanned return to operating room; WBC, white blood count.

Table 3 Associations between cardiac events and total length of hospital stay, readmission, and mortality

\begin{tabular}{|c|c|c|c|c|c|}
\hline Variables & $\begin{array}{l}\text { Cardiac events } \\
N=150\end{array}$ & $\begin{array}{l}\text { \% Cardiac } \\
\text { events }\end{array}$ & $\begin{array}{l}\text { No cardiac events } \\
N=2755\end{array}$ & $\begin{array}{l}\text { \% No cardiac } \\
\text { events }\end{array}$ & p-Value \\
\hline $\begin{array}{l}\text { Total length of hospital stay } \\
\text { in days (mean } \pm \text { SD) }\end{array}$ & \multicolumn{2}{|l|}{$13.2 \pm 14.7$} & \multicolumn{2}{|l|}{$8.3 \pm 9.32$} & $<0.01$ \\
\hline Readmission & 29 & $19.3 \%$ & 226 & $8.2 \%$ & $<0.01$ \\
\hline Mortality & 91 & $60.7 \%$ & 151 & $5.5 \%$ & $<0.01$ \\
\hline
\end{tabular}

Abbreviation: SD, standard deviation.

coronary artery stenosis resulting from vascular calcification and atherosclerosis, in addition to ischemia caused by decreased oxygen delivery, ${ }^{15}$ which may make them more susceptible to developing postoperative CEs. In fact, CEs have been reported concurrently as the largest single cause of mortality in dialysis patients. ${ }^{16-18}$ The finding that ASA $>3$ is independently associated with CEs is expected since other reports confirm a significant association between comorbidity and adverse postoperative outcomes. ${ }^{19-23}$

Findings of our study suggest that perioperative blood transfusions independently increase rates of post-TEVAR CEs; however, this association should be interpreted cautiously. In 
fact, even though some studies demonstrate an independent association between perioperative blood transfusion and adverse postoperative outcomes, ${ }^{24-27}$ it is worthwhile to note that patients who are transfused perioperatively, in general, tend to be sicker and have several comorbid conditions including anemia which independently increase their risks of postoperative adverse outcomes. ${ }^{28-31}$ Nonetheless, that PRBCs contain proinflammatory cytokines which increase systemic inflammatory response, and have been noted to adhere to vascular endothelium leading to reduced microvascular flow and tissue hypoxia ${ }^{25,32,33}$ demonstrate that emphasis should be placed on continuous cardiac monitoring of patients who undergo perioperative blood transfusions.

The effect of mechanical ventilation on cardiovascular performance is well-reported. ${ }^{34,35}$ Mechanical ventilation induces changes in intrapleural or intrathoracic pressure and lung volume and causes changes in atrial filling or preload, impedance to ventricular emptying or afterload, heart rate and myocardial contractility. ${ }^{34,35}$

Unplanned reintubation is an unexpected occurrence after surgery and usually represents a deteriorating postoperative course. Patients who undergo unplanned reintubation have been reported to have higher complication rates and mortality. ${ }^{36-38}$ Though reasons for unplanned reintubation for individual patients are not provided by the ACS-NSQIP database, indications usually range from emergency management of failed extubation, reintubations for emerging complications such as refractory hypotension or inability to protect airway, or emergency tracheostomy, ${ }^{4,36}$ all of which represent clinical deterioration.

Lastly, it is worthwhile to note that the benefits of preoperative laboratory assessments in the patients undergoing surgery have been discussed. ${ }^{39,40}$ In the present study leukocytosis was observed as an independent predictor of CEs after TEVAR. Preoperative leukocytosis may reflect an inflammatory response that might be associated with vascular injury and organ dysfunction, ${ }^{39,40}$ increasing the likelihood of CEs.

\section{Limitations}

The present study demonstrates that the cause of CEs following TEVAR is multifactorial. These results should be interpreted within the context of some limitations. First, the retrospective and observational nature of the study makes it difficult to accurately prove causation. Nonetheless, our multivariate analysis helped to control for multiple covariates. Second, the ACS-NSQIP database has its inherent limitations. The ACS-NSQIP only provides information on 30-day morbidity and mortality and does not elaborate on severity of some of the comorbid conditions. It is possible that some variables that were not significant in our study could be the predictors of post-TEVAR CEs in the long term. Similarly, the ACS-NSQIP database classifies cases based on CPT codes; hence, anatomical differences, procedural technique differences, and complication severity remain unclear. Additionally, though no statistical difference was observed in the incidence of post-TEVAR CEs between patients with aneurysm and dissection, the ACS-NSQIP database does not differentiate between Stanford Type A and Type B aortic dissections, making it difficult to account for any associations. In addition, the ACS-NSQIP database disproportionately represents data from large academic quality-seeking institutions due to the costs associated with participating and contributing to this database. ${ }^{41}$ Hence, a disparity of risk factors may exist at other nonparticipating institutions, making our results nongeneralizable. Finally, ACS-NSQIP database is also subject to recording errors even though trained individuals at each institution are assigned to input data. Despite these limitations, the ACS-NSQIP database is a validated database with high inter-rater reliability that provides accurate data and allows for understanding of postoperative patient outcomes. ${ }^{41,42}$

\section{Conclusions}

This is the first large multicenter study that provides a practical model to predict risk factors for CEs following TEVAR. Emergency surgery, ASA $>3$, dependent functional status, ventilator dependence prior to surgery, perioperative blood transfusions (preoperative and postoperative), preoperative leukocytosis, preoperative renal failure, postoperative prolonged mechanical ventilation, and postoperative unplanned reintubation are strong independent predictors of CEs following TEVAR. Recognizing these identified risk factors should allow for increased preventative approaches to decrease CEs and improve TEVAR outcomes. Future studies should prospectively evaluate these predictors to comprehensively inform surgeons on risk stratification for postTEVAR cardiac outcomes.

\section{Funding}

None.

\section{Conflict of Interest}

The authors declare no conflict of interest related to this article.

\section{Acknowledgments}

Study design: I.M.L., D.O.A.; Data acquisition: I.M.L., D.O.A.; manuscript preparation: D.O.A., I.M.L., P.P.; critical revision of manuscript: D.O.A., I.M.L., P.B.; and final approval of manuscript: D.O.A., I.M.L., and P.B. This study was presented at the 13th Annual Academic Surgical Congress, January 30 to February 1, 2018.

\section{References}

1 Ganapathi AM, Englum BR, Schechter MA, et al. Role of cardiac evaluation before thoracic endovascular aortic repair. J Vasc Surg 2014;60(05):1196-1203

2 Bub GL, Greenberg RK, Mastracci TM, et al. Perioperative cardiac events in endovascular repair of complex aortic aneurysms and association with preoperative studies. J Vasc Surg 2011;53(01): 21-27.e1, 2

3 Fink AS, Campbell DA Jr, Mentzer RM Jr, et al. The National Surgical Quality Improvement Program in non-veterans administration hospitals: initial demonstration of feasibility. Ann Surg 2002;236(03):344-353, discussion 353-354 
4 American College of Surgeons. User Guide for the 2015 ACS NSQIP Participant User Data File. Available at: https://www.facs.org/ /media/files/quality\%20programs/nsqip/nsqip_puf_user_guide_2015. ashx. Accessed March 15, 2018

5 Parmar CD, Torella F. Prediction of major adverse cardiac events in vascular surgery: are cardiac risk scores of any practical value? Vasc Endovascular Surg 2010;44(01):14-19

6 Bertges DJ, Goodney PP, Zhao Y, et al; Vascular Study Group of New England. The Vascular Study Group of New England Cardiac Risk Index (VSG-CRI) predicts cardiac complications more accurately than the Revised Cardiac Risk Index in vascular surgery patients. J Vasc Surg 2010;52(03):674-683, 683.e1-683.e3

7 Parker JD, Golledge J. Outcome of endovascular treatment of acute type B aortic dissection. Ann Thorac Surg 2008;86(05):1707-1712

8 Goodney PP, Travis L, Lucas FL, et al. Survival after open versus endovascular thoracic aortic aneurysm repair in an observational study of the Medicare population. Circulation 2011;124(24): 2661-2669

9 Davies JE. Endovascular repair of descending thoracic aortic aneurysm: review of literature. Semin Thorac Cardiovasc Surg 2009;21(04):341-346

10 Suzuki T, Mehta RH, Ince H, et al; International Registry of Aortic Dissection. Clinical profiles and outcomes of acute type B aortic dissection in the current era: lessons from the International Registry of Aortic Dissection (IRAD). Circulation 2003;108(Suppl 1):II312-II317

11 Christou NV, Morin JE. Host defense mechanisms in elective and emergency vascular surgery: predicting septic-related mortality. J Vasc Surg 1986;3(02):338-342

12 Scarborough JE, Bennett KM, Englum BR, Pappas TN, LagooDeenadayalan SA. The impact of functional dependency on outcomes after complex general and vascular surgery. Ann Surg 2015;261(03):432-437

13 Crawford RS, Cambria RP, Abularrage CJ, et al. Preoperative functional status predicts perioperative outcomes after infrainguinal bypass surgery. J Vasc Surg 2010;51(02):351-358, discussion 358-359

14 Nathan DP, Tang GL. The impact of chronic renal insufficiency on vascular surgery patient outcomes. Semin Vasc Surg 2014;27 (3-4):162-169

15 Hori D, Yamaguchi A, Adachi H. Coronary artery bypass surgery in end-stage renal disease patients. Ann Vasc Dis 2017;10(02):79-87

16 Herzog CA. Can we prevent sudden cardiac death in dialysis patients? Clin J Am Soc Nephrol 2007;2(03):410-412

17 Yotsueda R, Taniguchi M, Tanaka S, et al. Cardiothoracic ratio and allcause mortality and cardiovascular disease events in hemodialysis patients: the Q-Cohort study. Am J Kidney Dis 2017;70(01):84-92

18 Svensson LG, Crawford ES, Hess KR, Coselli JS, Safi HJ. Experience with 1509 patients undergoing thoracoabdominal aortic operations. J Vasc Surg 1993;17(02):357-368, discussion 368-370

19 Rudarakanchana N, Reeves BC, Bicknell CD, Heatley FM, Cheshire NJ, Powell JT. Treatment decisions for descending thoracic aneurysm: preferences for thoracic endovascular aneurysm repair or surveillance in a discrete choice experiment. Eur J Vasc Endovasc Surg 2014;48(01):13-22

20 Wiedemann D, Mahr S, Vadehra A, et al. Thoracic endovascular aortic repair in 300 patients: long-term results. Ann Thorac Surg 2013;95(05):1577-1583

21 Scali ST, Chang CK, Feezor RJ, et al. Preoperative prediction of mortality within 1 year after elective thoracic endovascular aortic aneurysm repair. J Vasc Surg 2012;56(05):1266-1272, discussion 1272-1273

22 Patterson B, Holt P, Nienaber C, Cambria R, Fairman R, Thompson $M$. Aortic pathology determines midterm outcome after endovascular repair of the thoracic aorta: report from the Medtronic Thoracic Endovascular Registry (MOTHER) database. Circulation 2013;127(01):24-32

23 Grabenwöger M, Alfonso F, Bachet J, et al; European Association for Cardio-Thoracic Surgery (EACTS); European Society of Cardiology (ESC); European Association of Percutaneous Cardiovascular Inter- ventions (EAPCI). Thoracic Endovascular Aortic Repair (TEVAR) for the treatment of aortic diseases: a position statement from the European Association for Cardio-Thoracic Surgery (EACTS) and the European Society of Cardiology (ESC), in collaboration with the European Association of Percutaneous Cardiovascular Interventions (EAPCI). Eur J Cardiothorac Surg 2012;42(01):17-24

24 Murthy T. Blood transfusion practices in sepsis. Indian J Anaesth 2014;58(05):643-646

25 O'Keeffe SD, Davenport DL, Minion DJ, Sorial EE, Endean ED, Xenos ES. Blood transfusion is associated with increased morbidity and mortality after lower extremity revascularization. J Vasc Surg 2010;51(03):616-621, 621.e1-621.e3

26 Bursi F, Barbieri A, Politi L, et al. Perioperative red blood cell transfusion and outcome in stable patients after elective major vascular surgery. Eur J Vasc Endovasc Surg 2009;37(03):311-318

27 Napolitano LM. Perioperative anemia. Surg Clin North Am 2005; 85(06):1215-1227, $\mathrm{x}$

28 Gupta PK, Sundaram A, Mactaggart JN, et al. Preoperative anemia is an independent predictor of postoperative mortality and adverse cardiac events in elderly patients undergoing elective vascular operations. Ann Surg 2013;258(06):1096-1102

29 Vincent JL, Baron JF, Reinhart K, et al; ABC (Anemia and Blood Transfusion in Critical Care) Investigators. Anemia and blood transfusion in critically ill patients. JAMA 2002;288(12):1499-1507

30 Gorla R, Tsagakis K, Horacek M, et al. Impact of preoperative anemia and postoperative hemoglobin drop on the incidence of acute kidney injury and in-hospital mortality in patients with type B acute aortic syndromes undergoing thoracic endovascular aortic repair. Vasc Endovascular Surg 2017;51(03):131-138

31 Ohtake H, Kimura K, Sanada J, Matsui O, Watanabe G. Risk factor analysis of thoracic endovascular repair using the Matsui-Kitamura stent graft for acute aortic emergencies in the descending thoracic aorta. J Vasc Surg 2010;52(06):1464-1470

32 Murphy GJ, Reeves BC, Rogers CA, Rizvi SI, Culliford L, Angelini GD. Increased mortality, postoperative morbidity, and cost after red blood cell transfusion in patients having cardiac surgery. Circulation 2007;116(22):2544-2552

33 Liumbruno G, Bennardello F, Lattanzio A, Piccoli P, Rossetti G. Recommendations for the transfusion of red blood cells. Blood Transfus 2009;7(01):49-64

34 Shekerdemian L, Bohn D. Cardiovascular effects of mechanical ventilation. Arch Dis Child 1999;80(05):475-480

35 Duke GJ. Cardiovascular effects of mechanical ventilation. Crit Care Resusc 1999;1(04):388-399

36 Beverly A, Brovman EY, Malapero RJ, Lekowski RW, Urman RD. Unplanned reintubation following cardiac surgery: incidence, timing, risk factors, and outcomes. J Cardiothorac Vasc Anesth 2016;30(06):1523-1529

37 Nafiu OO, Ramachandran SK, Ackwerh R, Tremper KK, Campbell DA $\mathrm{Jr}$, Stanley JC. Factors associated with and consequences of unplanned post-operative intubation in elderly vascular and general surgery patients. Eur J Anaesthesiol 2011;28(03):220-224

38 Ramachandran SK, Nafiu OO, Ghaferi A, Tremper KK, Shanks A, Kheterpal S. Independent predictors and outcomes of unanticipated early postoperative tracheal intubation after nonemergent, noncardiac surgery. Anesthesiology 2011;115(01):44-53

39 Mahmood E, Knio ZO, Mahmood F, et al. Preoperative asymptomatic leukocytosis and postoperative outcome in cardiac surgery patients. PLoS One 2017;12(09):e0182118

40 Lindner HA, Balaban Ü, Sturm T, Weiß C, Thiel M, SchneiderLindner V. An Algorithm for systemic inflammatory response syndrome criteria-based prediction of sepsis in a polytrauma cohort. Crit Care Med 2016;44(12):2199-2207

41 Alluri RK, Leland H, Heckmann N. Surgical research using national databases. Ann Transl Med 2016;4(20):393

42 Kim L, Mabry C, Klimberg VS. Quality of benchmarks for assessment of care will influence outcome. Ann Surg 2007;245(05):672-673 